\title{
THE WEAK TOPOLOGY OF A BANACH SPACE
}

\author{
BY \\ H. H. CORSON
}

1. Introduction. The purpose of this investigation is to find criteria or techniques which can be used to determine whether or not a given Banach space $B$ under its weak topology has any of the usual topological properties. By usual properties I mean those which appear in either J. L. Kelley's, or Bourbaki's, treatment of topology $\left({ }^{1}\right)$.

The past results of this kind have dealt, to a large extent, with compactness. For example, Eberlein and Smulyan have proved the equivalence of various types of weak compactness, and there is Banach's theorem which characterizes reflexivity in terms of the weak compactness of the unit sphere. The theorems in this paper were suggested by these, and I give answers, or partial answers, to the following two questions.

The first question is: What are the special properties of a Banach space $B$ which is generated by a weakly compact, or weakly $\sigma$-compact, subset? (A set is $\sigma$-compact if it is the union of countably many compact sets.) Only a partial answer is given to this question. Also, a conjecture is formulated at the end of this introduction. However, it is not difficult to see that $B$ is spanned by a weakly compact subset if and only if it is spanned by a weakly $\sigma$-compact subset. Hence only the latter case need be discussed.

The immediate consequences of past results are these. First, it follows from Banach's theorem on reflexivity and a category argument that $B$ is weakly $\sigma$-compact if and only if $B$ is reflexive. Second, $B$ has a weakly dense countable subset if and only if $B$ is separable under its norm.

In $\$ 2$ two special classes of Banach spaces which are spanned by weakly $\sigma$-compact subsets are studied. These are the spaces of continuous complex valued functions $C_{0}(X)$ which vanish at infinity on a locally compact metric space $X$, and the spaces $L_{1}$ of complex valued functions which are integrable with respect to some finite measure on some set. In the case of $C_{0}(X)$ it is proved that $C_{0}(X)$ is weakly Lindelöf. Although the same seems to be true for $L_{1}$, only this is proved: If $L_{1}$ is the space of Haar integrable functions on a compact group $G$, then $L_{1}$ is Lindelöf under the weak* topology, where $L_{1}$ is considered as a subset of the linear functionals on $C(G)\left({ }^{2}\right)$.

The second question is: When is $B$ under its weak topology topologically

Presented to the Society, January 29, 1960; received by the editors August 3, 1960.

(1) It has been pointed out to me by V. L. Klee that some of the problems considered here have been posed by M. Katětov. (See Colloq. Mathematicum (1951) p. 98, pp. 299-300.)

(2) I wish to thank R. K. Getoor for some helpful conversations on this part. 
complete? (That is, when is $B$ under its weak topology homeomorphic with a closed subset of a product of metric spaces? Or equivalently, when is there a complete uniformity for $B$ consistent with the weak topology?) It is known from a theorem of $A$. Weil that, for closed subsets of such spaces, countable compactness is equivalent to compactness, and Grothendieck has pointed out the important role that this plays in his extension of the Eberlein theorem which was referred to above. It is proved in $\S 3$ that $B$ is weakly topologically complete if and only if every function on $B^{*}$ which agrees with an element of $B$ on each countable subset of $B^{*}$ is, in fact, an element of $B$. This result is used in $\$ 4$ to prove that the space of bounded sequences modulo the null sequences is not weakly topologically complete.

These theorems seem to support the conjecture that: $B$ is spanned by a weakly compact subset if and only if $B$ is weakly Lindelöf. Furthermore, it will be seen from the results in $\S 3$ that the apparent concentration of interest on compactness is actually due in part to the fact that so many properties, such as normality and paracompactness, seem to occur only in the presence of compactness.

As a final application of these theorems, it is shown in $\$ 4$ that certain projections do not exist. The results of this paper are applicable because the existence of a projection implies a weak homeomorphism which is shown to be impossible.

2. The spaces $L_{1}$ and $C_{0}(X)$. Before stating the main lemma on which all the theorems of this section depend, it is necessary to introduce some notation and terminology.

Let $C_{0}(X)$ denote the continuous, complex valued functions which vanish at infinity on the locally compact space $X$. If $X$ is also a metric space, then it is known that $X=\cup\left\{X_{\gamma}: \gamma \in \Gamma\right\}$, where $X_{\gamma}$ is open and separable and $X_{\gamma} \cap X_{\beta}=\varnothing$ for $\gamma \neq \beta[11,6 \mathrm{~T}]$. Of course this decomposition of $X$ is usually not unique. However, for a particular decomposition, for $\Gamma_{1} \subset \Gamma$, and for $f \in C_{0}(X)$, let $f / \Gamma_{1}$ be the function which is equal to $f$ on $U\left\{X_{\gamma}: \gamma \in \Gamma_{1}\right\}$ and which is zero otherwise. (If $\Gamma_{1}$ has only one member $\gamma$, then $f /\{\gamma\}$ will be written $f / \gamma$.) Also $A / \Gamma_{1}=\left\{f / \Gamma_{1}: f \in A\right\}$, for $A \subset C_{0}(X)$. A subset $A \subset C_{0}(X)$ will be said to be almost invariant under projection if, for some decomposition $\left\{X_{\gamma}: \gamma \in \Gamma\right\}$, there is a collection $\left\{\Gamma_{\sigma}: \sigma \in \Sigma\right\}$ of countable subsets of $\Gamma$ such that: (P) Each countable subset of $\Gamma$ is contained in one of the $\Gamma_{\sigma}$, and $\Gamma_{\sigma_{1}} \subset \Gamma_{\sigma_{2}} \subset \cdots$ implies that $A / U\left\{\Gamma_{\sigma_{i}}: i=1,2, \cdots\right\} \subset A .\left(C_{0}(X)\right.$ has this property. Also, see Lenma 3.) One can check that, if one decomposition works for $A$, so will any other.

It is a familiar fact that $C_{0}(X)$ is a Banach space under the norm $\|f\|$ $=\sup \{|f(x)|: x \in X\}$. A subset $A$ of $C_{0}(X)$ will be called w-compact, $w$-Lindelöf, or $w$-normal if $A$ is compact, or Lindelöf, or normal, as a subset of $C_{0}(X)$ under its weak topology. Using this terminology, the principal lemma reads: 
Lemma 1. A ssume $X$ is a locally compact metric space. Then any $A \subset C_{0}(X)$ which is almost invariant under projection is w-Lindelöf.

Proof. To begin with, remember that the set $M(X)$ of continuous linear functionals $\mu$ on $C_{0}(X)$ can be represented as the finite, regular Borel (complex) measures on $X$ such that $\mu(f)=\int f d \mu$ for all $f \in C_{0}(X)[9, \S 56]$. Let $\left\{X_{\gamma}: \gamma \in \Gamma\right\}$ be a decomposition of $X$, fixed once and for all, such that $\left\{\Gamma_{\circ}: \sigma \in \Sigma\right\}$ has property (P). Then denote by $N$ the set of $\mu \in M$ such that the support of $\mu$ is contained in some $X_{\gamma}$ and $\|\mu\| \leqq 1$. (The former is equivalent here to the statement that for each $\mu \in N$ there is a $\gamma$ such that $\mu(f)=\mu(f / \gamma)$ for all $f \in C_{0}(X)$.)

Using this information, a slight, preliminary reduction will be made. Notice that, instead of all continuous linear functionals on $C_{0}(X)$, one may concentrate on $N$. This is true because, on the unit ball $S=\left\{f \in C_{0}(X):\|f\| \leqq 1\right\}$, the weak topology is the same as the weakest topology such that each $\mu \in N$ is continuous. The latter statement follows from an easy computation based on the fact that the set of linear combinations of elements of $N$ is norm dense in $M$. Also attention may be restricted to $A \cap S$ because $A$ is $w$ Lindelöf if $A \cap n S$ is $w$-Lindelöf for each integer $n$, and the argument for $A \cap n S$ is the same as the one for $A \cap S$. From now on, $A$ will be written for $A \cap S$.

Suppose that $\mathcal{U}$ is the cover of $A$ for which a countable subcover must be found. Let a fixed countable base $B=\left\{N_{8}: s=1,2, \ldots\right\}$ be chosen for the complex numbers. Then there is no loss of generality in supposing that for each element $U \in \mathcal{U}$ there are chosen $\mu_{i} \in N$ and $N_{s_{i}} \in ß, i=1, \cdots, n$, such that

$$
U=\left[\mu_{1}: s_{n}\right]=\left\{f \in A: \mu_{i}(f) \in N_{s_{i}}: i=1, \cdots, n\right\},
$$

since the collection of all such sets forms a base for the weak topology on $S$ as we have just seen.

Having made these simplifications, the proof will be interrupted in order to establish a special case of Lemma 1.

SPECIAL CASE. Let $\Gamma_{0}$ be a countable subset of $\Gamma$. Assume that $N=N_{\mathrm{a}} \in \mathbb{B}$ is jixed with $0 \in N$. Suppose that $v \subset \mathcal{C}$ has this property: If $\left[\mu_{1}: s_{n}\right] \in \mathcal{V}$, and $\mu_{i}$ has its support in $X_{\gamma}$ with $\gamma \in \in^{\prime} \Gamma_{0}$, then $N_{s_{i}} \supset N$. One can conclude from this that a countable subset of $V$ covers $U v$.

Proof of special case. Supposing that the statement above is false, an inductive proof will be used to get a contradiction. Pick a $V_{0}=\left[\mu_{1}: s_{n}\right] \in \mathcal{V}$. Let $\Gamma_{1}$ be the set of $\gamma \in \Gamma_{0}$ together with the $\gamma \in \Gamma \backslash \Gamma_{0}$ such that the support of one of the $\mu_{i}$ in the expression for $V$ is contained in $X_{\gamma} . \Gamma_{1} \backslash \Gamma_{0}$ is countable. Notice that some countable subset $V_{2}$ of $V$ covers $U V / \Gamma_{1}$. The latter is true because $C_{0}(X) / \Gamma_{1}$ is a separable Banach space, and each subspace is therefore Lindelöf. (Notice that, for each $V \in \mathcal{V}$, one has $f \in V$ if $f \in V / \Gamma_{1}$.)

Let $v_{1}=\left\{V_{0}\right\}$, and let $v_{2}$ be as above. Define $\Gamma_{2}$ as the set of $\gamma \in \Gamma_{1}$, 
together with the set of $\gamma \in \Gamma \backslash \Gamma_{1}$ such that the support of some $\mu$ in the expression for some $V \in v_{2}$ is contained in $X_{\gamma} . \Gamma_{2}$ is countable because $V_{2}$ is countable.

Using a similar argument at the $n$th stage of the induction, one may choose $\Gamma_{1}, \Gamma_{2}, \cdots$ and $v_{1}, v_{2}, \ldots$ such that $v_{i} \subset v$ is a countable cover of $U v / \Gamma_{i-1}$ and $\Gamma_{i}$ is the set of $\gamma \in \Gamma_{i-1}$, together with the $\gamma \in \Gamma \backslash \Gamma_{i-1}$ such that some $\mu$ in the expression for some $V \in V_{i}$ has its support in $X_{\gamma}$. Each $\Gamma_{i}$ is countable since each $v_{i}$ is.

Since the statement has been assumed to be false, there is an $f \in U \cup$ such that $f$ is not a member of any $V$ which is in a $V_{i}$. However, for each $i, f / \Gamma_{i-1}$ is a member of $V_{i} \in V_{i}$ for proper choice of $V_{i}$. Hence for some $\gamma_{i} \in \Gamma_{i}$ and some $\mu$ in the expression for $V_{i}, \mu(f) \in^{\prime} N$, since $f \in^{\prime} V_{i}$. Since $\|f\| \leqq 1$ and $\|\mu\| \leqq 1$, this implies that there is an $\epsilon>0$ independent of $i$ and $x_{i} \in X_{\gamma_{i}}$ for each $i$ such that $\left|f\left(x_{i}\right)\right|>\epsilon$. Hence $f$ does not vanish at infinity.

Now, back to the proof of Lemma 1 . Let $\Gamma_{0}$ be a countable subset of $\Gamma$ such that $\Gamma_{0}=\Gamma_{\sigma}$ for some $\sigma \in \Sigma$. (See the definition of almost invariant under projection.) Choose a fixed countable base $\Theta_{0}$ for $C(X) / \Gamma_{0}$. (This is a countable base for the norm topology. The weak topology will usually not have one.) For each $B \in B_{0}$, let $v_{B}$ be the set of all $U \in \mathcal{U}$ such that $U / \Gamma_{0} \supset B$. Let $v_{B, i}$ be the set of $V \in V_{B}$ such that $V / \gamma \supset N_{r_{i}}$ for each $\gamma \in \Gamma \backslash \Gamma_{0}$, where $\left\{N_{r_{i}}: i=1,2, \cdots\right\} \subset B$ is a fixed base at 0 for the complex numbers. By the special case there is a countable $\mathscr{W}_{B, i} \subset \mathcal{V}_{B, i}$ such that $W_{B, i}$ covers $U \mathcal{V}_{B, i}$.

Let $\Gamma_{1}$ contain the set of $\gamma \in \Gamma_{0}$, together with the $\gamma \in \Gamma \backslash \Gamma_{0}$ such that $X_{\gamma}$ contains the support of some $\mu$ in the expression for some $V \in U\left\{W_{B, i}: i=1,2, \cdots ; B \in B_{0}\right\}$. Also suppose that $\Gamma_{1}=\Gamma_{\sigma}$ for some $\sigma \in \Sigma$. $\Gamma_{1}$ is countable since each $W_{B, i}$ is, and the induction may be continued as above to find $B_{0}, B_{1}, \cdots$ and $\Gamma_{0}, \Gamma_{1}, \cdots$ with the following properties. (1) For each $n, \Gamma_{n-1} \subset \Gamma_{n}$, and $\Gamma_{n}$ is a countable subset of $\Gamma$ such that $\Gamma_{n}=\Gamma_{\text {. }}$ for some $\sigma \in \Sigma$. (2) $\bigotimes_{n}$ is a countable base for $C_{0}(X) / \Gamma_{n}$. (3) $\Gamma_{n}$ contains all $\gamma$ such that $X_{\gamma}$ contains the support of some $\mu$ in the expression for some $V \in U\left\{W_{B, i}: i=1,2, \cdots ; B \in \mathbb{B}_{n-1}\right\}$, where (4) $W_{B, i}$ is defined as above. That is, $\mathscr{W}_{B, i}$ covers $U \mathcal{V}_{B, i}$ and is countable. (Notice that $\mathcal{V}_{B, i}$ also depends on $n$, since $B$ does.)

Finally, one may see that $w$ (the set of $V$ such that $V$ is a member of some $W_{B, i}, i=1,2, \cdots$ and $\left.B \in B_{n}\right)$ is a countable subcover for $\mathcal{u}$. $W$ is countable since each $\mathscr{W}_{B, i}$ is countable; and $W_{\mathcal{C}}$. Hence it is only necessary to check that $W$ is a cover for $A$. This is where one utilizes the properties of the $\Gamma_{n}$ which follow from the assumption that $A$ is almost invariant under projection.

To show $W$ covers $A$, let $f \in A$. Then $g=f / \cup\left\{\Gamma_{n}: n=1,2, \cdots\right\} \in A$, and it suffices to show that $g$ is in some $U \in W$ because, by the construction of $W$, $f \in U \in W$ if $g$ is. Now $g \in U_{0}$ for some $U_{0} \in \mathcal{U}$. (Here we use the assumption that $\mathcal{U}$ is a cover.) Suppose $U_{0}=\left[\mu_{1}: s_{n}\right]$, then if the support of $\mu_{i}$ in the ex- 
pression for $U_{0}$ is in $X_{\gamma}$ for $\gamma \in^{\prime} \cup\left\{\Gamma_{n}: n=1,2, \cdots\right\}$ it follows that the corresponding $N_{8,}$ must be a neighborhood of 0 for the complex numbers. Hence one of the $N_{r_{j}}$ (the fixed collection of neighborhoods of 0 ) is contained in all the $N_{s_{i}}$ where $\mu_{i}$ is as above. Also one may pick an integer $p$ such that, if the support of a $\mu_{i}$ in the expression for $U_{0}$ is contained in $X_{\gamma}$ for $\gamma \in U\left\{\Gamma_{n}: n=1,2, \cdots\right\}$, then $\gamma \in \Gamma_{p}$. Let $B \in B_{p}$ such that $g \in B$ and $B \subset U_{0}$. Then $U_{0} \in \mathcal{V}_{B, j .}$. However, $W_{B, j}$ covers $U V_{B, j}$ and so $f \in U \in W_{B, j}$. This completes the proof.

Since $C_{0}(X)$ satisfies the hypothesis of Lemma 1 , Theorem 1 below is an immediate consequence.

TheOREM 1. If $X$ is locally compact and metrizable, then $C_{0}(X)$ is wLindelöf.

Perhaps this theorem is best translated into the language of the norm topology in the following way. (Recall that a collection of subsets of some set has c.i.p. if each countable subcollection of $K$ has a nonempty intersection.)

CoRollary 1. If $\mathfrak{K}$ is a collection of closed, convex subsets of $C_{0}(X)$ and if $\varkappa$ has c.i.p., then $\cap \kappa \neq \varnothing$.

Proof. Each $K \in \mathcal{K}$ is $w$-closed by Mazur's theorem [6, p. 22]. Hence, if $C_{0}(X)$ is not covered by the complements of countably many $K$, it is not covered by all their complements.

For locally compact $X$, it is not true that $C_{0}(X)$ is Lindelöf only if $X$ is metrizable. However, there is a partial converse to Theorem 1 which deals with the case that $X$ is a locally compact group. First, a lemma is needed which tells us that, for compact $X, C_{0}(X)$ has a large subset which is wdiscrete. (That is, it is $w$-closed in $C_{0}(X)$ and $w$-discrete in its relativized $w$-topology.)

Lemma 2. Let $X$ be a compact group. Then the set of characters of $X$ is wdiscrete in $C_{0}(X)=C(X)$. Also the set of real parts of the characters is w-discrete.

Proof. 'This follows from the orthogonality relations satisfied by the characters $[15$, p. 78$]$.

THEоRем 2. If $X$ is a locally compact group, then the following are equivalent.

(1) $C_{0}(X)$ is w-Lindelöf.

(2) $C_{0}(X)$ is w-normal.

(3) $X$ is metrizable.

Proof. (1) implies (2) since every Lindelöf space is normal $[11,5 \mathrm{Y}]$. (3) implies (1) by Theorem 1.

To prove that (2) implies (3), suppose that $X$ is not metrizable. Then it is easy to see that $X$ contains a compact subgroup $G$ which is not metrizable. 
(For instance, this follows from the assumption that $X$ is locally compact and the fact that a topological group $X$ is metrizable if and only if $X$ satisfies the first axiom of countability $[11$, p. 186].) If $G$ is not metrizable then it has an uncountable number of characters with distinct real parts. (This can be concluded from the above facts, the knowledge that the irreducible representations separate points in $G$, and the fact that different classes of irreducible representations have different characters $[15$, p.7 8].) Therefore Lemma 2 implies that $R(G)$, the set of real parts of the functions in $C(G)$, has an uncountable $w$-discrete subset. It follows that $R_{0}(X)$ has an uncountable $w$ discrete subset. Since $C_{0}(X)$, in its weak topology, is homeomorphic to $R_{0}(X) \times R_{0}(X)$, where each copy of $R_{0}(X)$ has the relativized weak topology, it remains to prove that this can not happen if $C_{0}(X)$ is w-normal. This follows from Lemma 7 in the next section, which completes the proof.

From what was said in the introduction one is led to conjecture that $L_{1}$ is also w-Lindelö, where $L_{1}$ denotes the Banach space of complex valued functions on a set $G$ which are integrable with respect to some finite measure $\nu$. (In fact, it is easy to see that $L_{1}$ is spanned by a $\sigma$-compact subset.) Although I have been unable to prove this conjecture, the next theorem shows that $L_{1}$ is Lindelof in a natural topology which is weaker (coarser) than the weak topology (for a special class of $L_{1}$ ). However, first let us recall some definitions.

If $G$ is compact, then $M(G)$ the finite, regular Borel measures on $G$ is the adjoint space of $C(G)$, as has already been noted. By the weak* topology on $M(G)$, one means the weakest topology on $M(G)$ such that each $f \in C(G)$ is continuous, where $f$ is now considered as a linear functional on $M(G)$. Theorem 3 concerns the measures $\mu$ which are absolutely continuous with respect to Haar measure $\nu$. That is, $\nu(E)=0$ implies $\mu(E)=0$ for $E \subset G$. (For Haar measure, see $[9$, Chapter XI].)

TheOREM 3. Let $G$ be a compact group. Then the collection of measures which are absolutely continuous with respect to Haar measure is weak* Lindelöf.

Proof. It is known that the set of linear combinations of the entries of the irreducible, unitary representations is uniformly dense in $C(G)[15$, p. 78]. It then follows from an elementary calculation that, on any sphere $S$ of $M(G)$, the weak* topology is the same as the weakest such that each such entry is continuous. For convenience, suppose $S$ is the unit sphere of $M(G)$.

The set of measures in $S$ which are absolutely continuous with respect to Haar measure corresponds, by a mapping which preserves norm, to the unit sphere $T$ of $L_{1}[9, \S 32]$. The topology on $T$ which is induced by the characters is the same as the weak topology on the collection $T^{-}$of Fourier transforms of elements of $T$, where $T^{-}$is considered as a subset of $C_{0}\left(G^{-}\right)$and $G^{-}$is the set of entries of a fixed total set of unitary representations of $G$.

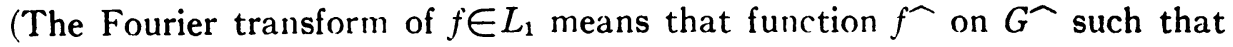


$f^{\curvearrowleft}(m)=\int f \bar{m} d \nu, m \in G^{-}$.) From these considerations one sees that it only remains to show that $T^{\sim}$ is almost invariant under projection.

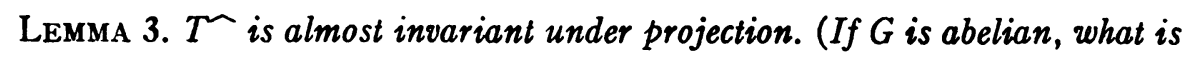
proved is this: $f \in L_{1}, F^{\wedge}$ a countable subgroup of $G^{\wedge}$ implies that there is a $g \in L_{1}$ such that $f^{\wedge}(\chi)=g^{\wedge}(\chi)$ if $\chi \in F^{\wedge}$ and $g(\chi)=0$ for $\chi \in F^{\prime}$.)

Proof. Assume that $H^{-}$is a countable subset of $G^{\frown}$. If $m_{i} \in H^{-}$, let $R_{i}$ be the unitary representation for which $m_{i}$ is an entry, and let $F_{i}$ be the kernel of $R_{i}$. Define $F$ as $\cap\left\{F_{i}: i=1,2, \cdots\right\}$. Since $F$ is a compact subgroup of the compact group $G$, one may choose an invariant measure $\alpha$ on $F$ and an invariant measure $\beta$ on $G / F$ such that, for all $f \in L_{1}, \int T f d \beta=\int f d \nu$ where $T f(x)$ $=\int f(x y) d \alpha(y)\left[15\right.$, p. 45]. Let $(G / F)^{-}$be the set of all $m \in G^{\wedge}$ such that $F$ is contained in the kernel of the representation for which $m$ is an entry. For $f \in L_{1}$, one may consider $T f$ to be a function in $L_{1}$ of $G$.

We wish to show first that either $m \in(G / F)^{-}$or $T m=0$. However, note that, by $\left[15\right.$, p. 76] $G^{-}$is an orthogonal basis for $L_{2}$ of $G$ and $(G / F)^{-}$is an orthogonal basis for $L_{2}$ of $G / F$, the latter assertion being true because $F$ is a normal subgroup of $G$. Therefore, it will be sufficient to prove that $T$ is an orthogonal projection in $L_{2}$ of $G$. Since $T$ is clearly a projection, it is sufficient to prove that $T$ is self adjoint, and this may be verified by using the Fubini theorem and the fact that $\alpha$ and $\beta$ are invariant.

Using the fact that either $m \in(G / F)^{-}$or $T m=0$, it is easy to verify that, for $f \in L_{1}$ of $G, T f(m)=0$ if $m \in^{\prime}(G / F)^{-}$while $T f(m)=f(m)$ when $m \in(G / F)$. Therefore let $\left\{\Gamma_{\sigma}: \sigma \in \Sigma\right\}$ be the set of all countable $(G / F)$ for $F$ a normal closed subgroup of $G$. By the above computation each countable subset of $G^{-}$is contained in some $\Gamma_{\sigma}, \sigma \in \Sigma$. To complete the proof, therefore, it is sufficient to show that, if $\Gamma_{\sigma_{1}} \subset \Gamma_{\sigma_{2}} \subset \cdots, \sigma_{i} \in \Sigma$, then $U_{i} \Gamma_{\sigma_{i}}=\Gamma_{\sigma}$ for some $\sigma \in \Sigma$. This may be verified using the compactness of $G$ and the fact that the range of each representation has no small subgroups. This completes the proof of Lemma 3 and, consequently, of Theorem 3.

The following corollary gives some idea of what Theorem 3 implies about $L_{1}$ in the norm topology. Also, compare it with Corollary 1 above.

Corollary 2. If a collection $s$ of closed spheres in $L_{1}$ has c.i.p., then $\cap s \neq \varnothing$.

Proof. $L_{1}$ is isometric to the collection referred to in the statement of Theorem 3, as noted in the proof of that theorem. Hence, each closed sphere in $L_{1}$ is weak* closed, and the proof used for Corollary 1 applies.

REMARKs. (1) I do not know if $w$-Lindelof is implied by the property in Corollary 1, nor the answer to the analogous question suggested by Corollary 2.

(2) It follows from a category argument, together with the Krein-Milman theorem, that most $L_{1}$ are not $\sigma$-compact in any locally convex topology which is weaker than the norm topology and for which the unit sphere is closed $[6$, p. 81, (5) (e)]. 
(3) Although the converse of Theorem 1 is not true, some information in this direction can be derived from Theorem 2, even for spaces which are not groups. As an example of how this can be done, see the proof of Example 1, $\$ 4$.

3. General theorems. The material in this section is motivated by three known facts. First, a (real) Banach space in its weak topology is homeomorphic in a natural way to a dense subset of a product of copies of the real numbers $[6$, p. 6]. Second, it is a closed subspace of a $R(X)$ for compact $X$, where $R(X)$ is given the topology of pointwise convergence. (Because the unit sphere of the adjoint of a Banach space is $w^{*}$-compact $[6$, p. 19].) Third, completeness is an important consideration in connection with Eberlein's theorem on w-compact subsets [8]. In the next lemma a simple fact is isolated which allows us to extend two theorems on product spaces so that they apply to dense subsets, and consequently to the weak topology.

Lemma 4. Let $A$ be a dense subset of a topological space $B$. If $\left\{U_{\gamma}: \gamma \in \Gamma\right\}$ is a disjoint collection of open sets in $A$, then there is a disjoint collection of open sets $\left\{V_{\gamma}: \gamma \in \Gamma\right\}$ in $B$ such that $V_{\gamma} \supset U_{\gamma}$ for each $\gamma \in \Gamma$.

Proof. For each $\gamma \in \Gamma$, pick any open $V_{\gamma} \subset B$ such that $U_{\gamma}=V_{\gamma} \cap A$. The collection of $V_{\gamma}$ has the required properties since $A$ is dense in $B$.

Before going on, a warning must be given here. The reader will want to check that Lemmas 5, 6, 7, 8 and 9 are valid for any dense subspace of a product of real lines. This is used in the proof of Theorem 4. The proofs of these generalizations are exactly the same as the proofs of the corresponding lemmas.

Now, recall that a topological space $B$ is paracompact if each open cover $\mathcal{u}$ of $B$ has a locally finite refinement $v$. (That is, each $V \in \mathcal{v}$ is contained in some $U \in \mathcal{U}$, and for $x \in B$ there is a neighborhood $N$ of $x$ such that only a finite number of the $V$ meet $N$.) It is known that a Lindelöf space is necessarily paracompact.

\section{LEMMA 5. If a Banach space $B$ is w-paracompact, then $B$ is w-Lindelöf.}

Proof. It is known that a paracompact space is Lindelöf if every metric space which is a continuous image of it is separable. (For instance, this follows immediately from [4].) This condition will obviously be satisfied if each disjoint collection of open sets is countable. This is known to be true for a product of copies of the real line [14], and hence it is also true for $B$ in the weak topology by Lemma 4.

The next few lemmas give an indication of how near to being w-paracompact certain $B$ are. (See $[11$, Chapter 5] for definitions, as well as proofs that paracompact spaces have the properties referred to in these lemmas.)

LEMMA 6. Let $E$ denote a w-dense subset of a Banach space $B$ under the weak topology. Then the collection of all the closed neighborhoods of the diagonal $\Delta$ in $E \times E$ is a uniformity for $E$ (compatible with the weak topology). 
Proof. This proof is similar to the proof of [5, Theorem 3]. To simplify notation, the proof below is given for $E=B$, but the more general statement follows similarly.

Let $F$ be a closed neighborhood of $\Delta$. Denote by $B^{* f}$ the space of all linear functionals (continuous or not) on $B^{*}$, the adjoint of $B$, where $B^{* f}$ is given the topology of pointwise convergence. If $U_{1}$ is the interior of $F$ and $U_{2}$ is the complement of $F$, then Lemma 4 implies that there are $V_{i} \supset U_{i}(i=1,2)$, with $V_{1} \cap V_{2}=\varnothing$ and each $V_{i}$ open in $B^{* f}$. It is well known $[6$, p. 12] that $B^{* t}$ can be considered as the space of all functions $\mathfrak{F}\left(E^{*}\right)$ on a maximal linearly independent subset $E^{*}$ of $B^{*}$. Hence if $s E^{*}$ is the topological sum of two copies of $E^{*}$, then $B^{*+} \times B^{*+}$ is the same as $\mathfrak{F}\left(s E^{*}\right)$. Since $V_{1} \cap V_{2}=\varnothing$, it is a consequence of [2] that there is a countable subset $K^{*} \subset E^{*}$ with the property: The restrictions $V_{i} / s K^{*}$ of the $V_{i}$ (or the natural projections, in another terminology) are disjoint (open) sets in $\mathfrak{F}\left(s K^{*}\right)$, the space of all functions on $K^{*}$ under the topology of pointwise convergence. However, the collection of all the neighborhoods of the diagonal in $\mathfrak{F}\left(s K^{*}\right)=\mathfrak{F}\left(K^{*}\right) \times \mathfrak{F}\left(K^{*}\right)$ is a uniformity for $\mathfrak{F}\left(K^{*}\right)$ because $\mathfrak{F}\left(K^{*}\right)$ is metrizable $[11$, p. 125$]$ and $V_{1} / s K^{*}$ is in this collection. It follows, as in [5], that $F$ is in the strongest (largest) uniformity compatible with the topology for $B$.

Let $E^{n}$ denote the topological product of $n$ copies of $E$.

LEMMA 7. If $E$ is a w-dense subset of $B$ and if $E^{2}$ is normal, then $E$ has no uncountable w-discrete subset.

(NotE. A paracompact topological space need not have the latter property, but a Lindelöf space always does.)

Proof. Suppose $E^{2}$ is normal, and $\left\{x_{\gamma}: \gamma \in \Gamma\right\}$ is a $w$-discrete subset of $E$. Then Lemma 6 implies that the collection of all the neighborhoods of the diagonal in $E^{2}$ is a uniformity for $E$. Under these conditions on $E$, it is proved in [3] that $E$ is collectionwise normal (see [1] for this term). In our case this implies that there is a disjoint collection $\left\{U_{\gamma}: \gamma \in \Gamma\right\}$ of open subsets of $E$ such that $x_{\gamma} \in U_{\gamma}$ for $\gamma \in \Gamma$. Hence $\Gamma$ can not be uncountable, as we have seen in the proof of Lemma 5.

The next sequence of results explain the introduction of the w-discrete subspaces. (Also, notice that $w$-discrete subspaces were an important idea in the proof of Theorem 2.) It will be seen how they enter into arguments concerning completeness. Here completeness does not refer to the fact that a Banach space is complete, although this is also important in this connection, but to the topological completeness of certain subsets of $B$ in the weak topology. (See the introduction for a definition of topologically complete.) Since this term is sometimes used for various other properties, I will call such an $E$ which is $w$-dense in $B$ real compact. This also might cause some slight confusion, since the assertion that $E$ is real compact ordinarily means that $E$ is complete in the uniformity $v$ generated by continuous real valued functions on $E-w e r e$ it not for the following result. 
LEMMA 8. $A$ w-dense subspace $E$ of $B$ is w-topologically complete if and only if $E$ is w-real compact.

Proof. Lemma 8 is true in general, except for a mild cardinality restriction [13]. In this case, however, suppose $E$ is topologically complete. It is not difficult to verify that it is sufficient to prove that each metric space which is the continuous image of $E$ is separable, since a separable metric space is real compact [7]. This has already been noted in the proof of Lemma 5. The converse is obvious from the definitions, hence the proof is complete.

Lemma 9 will characterize the $B$ which are $w$-real compact in terms of the following notions. On $B^{*}$, the adjoint of $B$, let the $\aleph_{0} w^{*}$-topology be the strongest (finest) which agrees with the weak* topology on $w^{*}$-separable subspaces of $B^{*}$. Then in $B^{* *}$ let $\aleph_{0} B$ be all the $\aleph_{0} w^{*}$-continuous functions.

LEMMA 9. $B$ is w-real compact if and only if $B=\aleph_{0} B$.

Proof. Suppose $B$ is $w$-real compact and $X \in B^{* *}$. For each countable subset $K \subset B^{*}$, let $F(K)$ be the set of $x \in B$ such that $f(x)=X(f)$ for all $f \in K$. If $\mathcal{F}$ is the collection of all such $F(k)$, it will suffice to show $\mathcal{F}$ is a filter which is Cauchy in the uniformity $v$ defined by the $w$-continuous real functions on $B$. In fact $\mathcal{F}$ is obviously a filter, and if $\mathcal{F}$ is Cauchy for $\mathcal{V}$ and $x_{0}$ is its limit, then one easily verifies that $X=x_{0} \in B$.

To show that $\mathcal{F}$ is Cauchy for $\mathcal{V}$, let $\phi$ be a $w$-continuous real function on $B$. For each rational number $r$, let $L_{r}=\{x \in B: \phi(x)<r\}$. Similarly, let $U_{r}=\{x \in B: \phi(x)>r\}$. Lemma 4 is used to find $V\left(L_{r}\right)$ and $V\left(U_{r}\right)$, disjoint $w^{*}$-open sets in $B^{* *}$ such that $V\left(L_{r}\right) \supset L_{r}$ and $V\left(U_{r}\right) \supset U_{r}$. By [2] it is possible to find a countable subset $K_{r} \subset B^{*}$ such that $V\left(L_{r}\right) / K_{r}$ is disjoint from $V\left(U_{r}\right) / K_{r}$. Let $K_{0}=U\left\{K_{r}: r\right.$ rational $\}$. It is easy to see that there is a real function $g$ defined on $B / K_{0}$ such that $\phi=g \pi$, where $\pi$ is the natural projection from $B$ to $B / K_{0}$. Hence $F\left(K_{0}\right)$ is $\phi$-small, and $\mathcal{F}$ is $\mathcal{V}$-Cauchy.

The converse is a consequence of two facts which are left for the reader to verify. (1) $\aleph_{0} B$ (in the $w^{*}$-topology) is the inverse limit of $\{B / K: K$ countable, $\left.K \subset B^{*}\right\}$ under the natural mappings, where each $B / K$ has the topology of pointwise convergence on $K$. (2) Each $B / K$ is separable metric in the above topology. From the definition of the inverse limit and (1), $\boldsymbol{N}_{0}(B)$ is a closed subset of the product of the separable metric $B / K$. Hence. $\aleph_{0}(B)$ is $w^{*}$-real compact (see [7]), and so is $B$ if $B=\boldsymbol{N}_{0}(B)$.

REMARK. In any case $\boldsymbol{N}_{0} B$ is the real compactification of $B$. (See [7] for this term.) This follows from the above proof as soon as one checks that $g$ is continuous - which is not immediate, since $\pi$ need not be a decomposition mapping.

The last of this sequence of lemmas treats a condition which implies that $B$ is $w$-real compact. This condition concerns the unit sphere $S^{*}$ in $B^{*}$. However, let $X$ be any topological space. Define seq $X$ to be $X$ under the stronger 
topology derived from the old one by: $E \subset$ seq $X$ is closed if each point $x$ which is in the closure of some countable subset of $E$, is in $E$. To see how this property is used, suppose that seq $S^{*}=S^{*}$, where $S^{*}$ has the $w^{*}$-topology. It is clear that, if $F \in \aleph_{0} B$, then $F / S^{*}$ is $w^{*}$-continuous on $S^{*}$. Hence, $F$ is $w^{*}$-continuous $[6$, p. 43], and is in $B[6$, p. 18] .

Recall that $R(X)$ is the collection of continuous real functions on $X$.

Lemma 10. Let $X$ be a compact space, and let $A$ be uniformly dense in $R(X)$. If seq $X \neq X$, then there is an uncountable subset of $A$ which is closed in $R(X)$ and discrete in the topology of pointwise convergence.

Proof. This proof is an extension of the proofs of Eberlein and Grothendieck to situations where one does not have a certain property concerning countability.

Suppose $y_{0} \in E^{-}$(the closure of $E$ in $X$ ), but $y_{0} \in K^{-}$for any countable $K \subset E$. Let $x_{0}$ be some element in $E^{\sim}$ (the closure of $E$ in seq $X$ ). Let $f_{0} \in A$ be chosen with the property that $\left|f_{0}\left(x_{0}\right)-f_{0}\left(y_{0}\right)\right| \geqq 1$. This is possible since $A$ is uniformly dense in $R(X)$.

By induction one may choose, for each countable ordinal $\alpha, f_{\alpha} \in A$ and $x_{\alpha} \subset E$ with these properties. First, $\left|f_{\alpha}\left(x_{\gamma}\right)-f_{\alpha}\left(y_{0}\right)\right| \geqq 1$ for $\gamma \leqq \alpha$, and second $\left|f_{\gamma}\left(x_{\alpha+1}\right)-f_{\gamma}\left(y_{0}\right)\right| \leqq 1 / 4$ for $\gamma \leqq \alpha$. In fact, the only thing to prove is that, for any countable ordinal $\alpha$,

$$
\text { (i) }\left\{x \in E:\left|f_{\gamma}(x)-f_{\gamma}\left(y_{0}\right)\right| \leqq 1 / 4 \text { for } \gamma \leqq \alpha\right\} \neq \varnothing \text {. }
$$

To prove this, first notice that seq $X$ is countably compact, and so $E^{\sim}$ is countably compact. Also, $f_{\gamma}$ is continuous in seq $X$ for each $\gamma<\alpha$, since it is continuous in $X$. Hence (i) must be true because, otherwise, there would be a countable cover for $E^{\sim}$ which had no finite subcover.

Now one can prove that the set of $f_{\alpha}$ is the discrete set whose existence is asserted in the lemma. To do this, suppose that $g$ is in the pointwise closure of $\left\{f_{\alpha}: \alpha<\beta\right\}$, and suppose that $\beta$ is the first ordinal with that property. (One may assume that $\beta$ is a limit ordinal, since the other case is trivial.) If $z_{\beta}$ is a cluster point of the net $\left\{x_{\alpha}: \alpha<\beta\right\}$, one has a contradiction because of the next sequence of inequalities. First, $\left|f_{\gamma}\left(z_{\beta}\right)-f_{\gamma}\left(y_{0}\right)\right| \leqq 1 / 4$, since $\left|f_{\gamma}\left(x_{\alpha}\right)-f_{\gamma}\left(y_{0}\right)\right| \leqq 1 / 4$ for $\gamma<\alpha$ and $f_{\gamma}$ is continuous. Next, $\left|f_{\gamma}\left(x_{\alpha}\right)-f_{\gamma}\left(z_{\beta}\right)\right|$ $\geqq 3 / 4$ for $\gamma \geqq \alpha$, since

$$
\left|f_{\gamma}\left(x_{\alpha}\right)-f_{\gamma}\left(z_{\beta}\right)\right| \geqq\left|f_{\gamma}\left(x_{\alpha}\right)-f_{\gamma}\left(y_{0}\right)\right|-\mid f_{\gamma}\left(y_{0}\right)-\left(f_{\gamma}\left(z_{\beta}\right) \mid \geqq 3 / 4 .\right.
$$

Finally, $\left|g\left(x_{\alpha}\right)-g\left(z_{\beta}\right)\right| \geqq 3 / 4$, since $g$ is in the closure of $\left\{f_{\gamma}: \gamma<\beta\right\}$ and since $\beta$ is minimal. However, this is impossible because $g$ was assumed to be continuous.

These lemmas imply the next theorem. In the statement of this theorem, $B^{n}$ means the product of $n$ copies of $B$. Notice that the weak topology of $B^{n}$ 
is the same as the product topology derived from giving each copy of $B$ the weak topology.

THEOREM 4. If $B^{n}$ is w-normal for each integer $n$, then $B$ is w-real compact, and the collection of all the neighborhoods of the diagonal in $B^{2}$ is an admissible uniformity for $B$ in its w-topology. If $B$ is w-paracompact, then $B$ is w-Lindelof.

Proof. $B$ in the w-topology can be considered as a closed subspace of $C\left(S^{*}\right)$, where $S^{*}$ is the unit sphere of $B^{*}$ under the $w^{*}$-topology and $C\left(S^{*}\right)$ has the topology of pointwise convergence. Let $A$ be the algebra generated (algebraically) in $C\left(S^{*}\right)$ by $B$ and the constant functions. It will be proved that $A$ has no uncountable discrete subspace. Assuming this, then seq $S^{*}$ $=S^{*}$ by Lemma 10 . By the remarks preceding Lemma $10, B=\aleph_{0} B$, and $B$ is $w$-real compact by Lemma 9 . To prove the missing step, notice that $B^{n}$ has no uncountable $w$-discrete subset, because of Lemma 7 and the fact that $B^{2 n}$ is normal. $A$ is the union of continuous images of a countable number of sets of the form $B^{n} \times R^{n+1}$. It is not hard to check that the sets of the latter form have no uncountable discrete subsets if $B^{n}$ does not, and this property is inherited by continuous images and closed under countable unions. Hence $A$ has the property.

The rest of the first sentence of the theorem is a consequence of Lemma 6 and the assumption that $B^{2}$ is normal. The last part of the theorem is a repetition of Lemma 5.

REMARKS. (1) The reader deserves some explanation for the claims for Theorem 4 that have been made in the introduction-which are, that it shows that almost any type of normality for the w-topology of $B$ will, in most cases, imply that $B$ is $w$-Lindelöf. There are two gaps. First, the assumption that $B^{n}$ is $w$-normal would follow from the hypothesis that $B$ is $w$ normal if one knew that, for any Banach space $E, E^{2}$ is isomorphic to $E$. This was long an unsolved problem of Banach $[10$, p. 5]. Although it is now solved in the negative, it is still not known if $E^{2}$ and $E$ are $w$-homeomorphic. Second, it is not true that, if all the neighborhoods of the diagonal are a complete uniformity for an arbitrary space $X$, then $X$ is paracompact; but this was a conjecture of Kelley which was only recently shown to be false [5].

4. Examples. Let $m=m(J)$ be the Banach space of bounded sequences, that is, bounded complex functions on the integers $J$. Let $c_{0}=C_{0}(J)$, the set of $x \in m$ which vanish at infinity.

EXAMPLE 1. (i) The space $m$ is $w$-real compact, but not $w$-normal (hence not $w$-Lindelöf).

(ii) However, $m / c_{0}$ is not w-real compact (hence there is no projection of $m$ on to $c_{0}[6$, p. 96]).

(iii) If $s^{*}$ is the unit sphere of $m^{*}$ in the $w^{*}$-topology, then seq $s^{*} \neq s^{*}$ (however, $\aleph_{0} m=m$ by (i)). 
Proof. (i) Since $P$, the product of $\aleph_{1}$ copies of the unit circle, is separable, one can suppose that $J$ is the dense subset of $P$. Hence $C(P)$ is a closed subspace of $m$, and $C(P)$ is not $w$-normal because $P$ is not metrizable. (See Theorem 2.) However, $m$ is $w$-real compact because $s^{*}$ is $w^{*}$-separable $[6$, p. 78], and hence $\boldsymbol{N}_{0}(m)=m$. (See Lemma 9.)

(ii) For each countable ordinal $\alpha$, let $\Gamma_{\alpha}$ be an infinite subset of $J$ such that $\Gamma_{\alpha} \subset \Gamma_{\beta} \cup F_{\alpha \beta}$ for $\alpha<\beta$, where $F_{\alpha \beta}$ is a finite subset of $J$ depending on $\alpha$ and $\beta$, and $\Gamma_{\beta} \backslash \Gamma_{\alpha}$ is infinite. (It is known that this is possible [12].) For each $\alpha$, let $x_{\alpha}$ be 1 on $\Gamma_{\alpha}$ and 0 otherwise. It will be shown that the net $x_{\alpha} w^{*}$. converges to a function $F \in \aleph_{0} m$. To do this, recall that each $f \in m^{*}$ can be considered as a function of bounded variation on the subsets of $J$ such that, for characteristic functions such as $x_{\alpha}, f\left(x_{\alpha}\right)=f\left(\Gamma_{\alpha}\right)[6$, p. 30]. Hence one can check that each $f \in\left(m / c_{0}\right)^{*}$ can be thought of as an $f \in m^{*}$ such that $f(\Gamma)=0$ for all finite subsets of $J$. Since $f \in m^{*}$ is finitely additive, $f\left(x_{\alpha}\right)=f\left(\Gamma_{\alpha}\right) \leqq f\left(\Gamma_{\beta}\right)$ $=f\left(x_{\beta}\right)$ for $\alpha<\beta$ and $f \in\left(m / c_{0}\right) *$. It follows from the properties of the countable ordinals that $f$ is eventually constant, say $F(f)$. The function $F$ so defined is a member of $\aleph_{0} m$ by the same argument, since for any countable set $f_{1}, f_{2}, \cdots$ in $m^{*}, F\left(f_{i}\right)=f_{i}\left(x_{\alpha}\right)$ for all $i$ if $\alpha$ is chosen large enough. However, $F \in^{\prime} m$ as will be shown.

For each ordinal $\alpha$, let $U_{\alpha}$ be a nontrivial ultrafilter in $J$ which is eventually contained in $\Gamma_{\alpha+1} \backslash \Gamma_{\alpha}$. Let $f_{\alpha} \in\left(m / c_{0}\right)^{*}$ be defined by $f_{\alpha}(\Gamma)=1$ if $U_{\alpha}$ is eventually in $\Gamma$, and $f_{\alpha}(\Gamma)=0$ otherwise. Let $g$ be any $w^{*}$-cluster point of the net $f_{\boldsymbol{\alpha}}$. Then

$$
F(g)=\lim _{\beta} g\left(x_{\beta}\right)=\lim _{\beta} \lim _{\alpha} f_{\alpha}\left(x_{\beta}\right)=\lim _{\beta} \lim _{\alpha} f_{\alpha}\left(\Gamma_{\beta}\right)=0 .
$$

On the other hand, if $F$ were $w^{*}$-continuous,

$$
F(g)=\lim _{\alpha} F\left(f_{\alpha}\right)=\lim _{\alpha} \lim _{\beta} f_{\alpha}\left(x_{\beta}\right)=\lim _{\alpha} \lim _{\beta} f_{\alpha}\left(\Gamma_{\beta}\right)=1 .
$$

Hence $F \in^{\prime} m$, and the proof of (ii) is complete.

(iii) In the proof of (ii), $g$ is in the closure of $\left\{f_{\alpha}: \alpha<\Omega\right\}$, but $g$ is not in the closure of any countable subset of this set.

Example 2. As we have seen, it would be interesting to know if $B \times B$ is $w$-normal whenever $B$ is w-normal. I have not been able even to find two Banach spaces $C$ and $c_{0}$ such that both $C$ and $c_{0}$ are $w$-normal, but $C \times c_{0}$ is not. However, the space $C_{r}$ of complex valued functions on the unit interval $I$ which are continuous on the right and which have a finite limit on the left gives rise to an example which is close to the latter situation. Let $C$ be the continuous functions on $I$, and let $c_{0}=C_{r} / C$.

(i) The spaces $C$ and $c_{0}$ are w-Lindelöf (hence w-normal), but $C_{r}$ is not wnormal (hence not w-Lindelöf).

(ii) There is no projection of $C_{r}$ onto $C$. 
Proof of (i). $C$ is $w$-Lindelöf since it is separable. $c_{0}$ is $w$-Lindelöf by Lemma 1 of $\$ 2$ because it is isomorphic to the space $B$ of all functions on $I$ which vanish at infinity - where $I$ is given the discrete topology. Let us check this last assertion. If $t$ is a point where $x$ is not continuous, then call $x(t)$ $-x\left(t^{-}\right)$a jump of $x$. It is clear that each coset $x+C$ of $c_{0}$ is uniquely determined by the jumps of any function in $x+C$. For each $x+C$, let $\phi[x+C]$ be the function on $I$ defined by $\phi[x+C](t)=x(t)-x\left(t^{-}\right)$. It is easy to see that each $\phi[x+C] \in B$. Moreover, $\|x+C\|=1 / 2\|\phi[x+C]\|$ as may be easily verified. Since $c_{0}$ is complete $[6, \dot{p} .25]$, since the collection of $\phi[x+C]$ contains all functions on $I$ which vanish off finite sets, and since this collection is dense in $B, \phi$ is an isomorphism between $B$ and $c_{0}$.

To show that $C_{r}$ is not $w$-normal, define for each $t \in I$ the function $x_{t}$ by

$$
x_{t}(s)= \begin{cases}0 & \text { for } s<t, \\ 1 & \text { for } t \leqq s .\end{cases}
$$

The collection $D=\left\{x_{t}: t \in I\right\}$ is a closed, discrete subspace of $C_{r}$, and the cardinality of $D$ is $2{ }^{N_{0}}$. If $C_{r}$ were $w$-normal, there would be $2{ }^{2 N_{0}} w_{\text {-continuous }}$ real-functions on $C_{r}$, since every function on $D$ could be extended continuously to $C_{r}$ by using Urysohn's lemma. On the other hand, one can prove that there are at most $2{ }^{N_{0}}$ w-continuous real functions on $C_{r}$. To do this, notice that the adjoint of $c_{0}$ is of cardinality $2^{N_{0}}$ and the adjoint of $C$ is of cardinality $2^{N_{0}}$. Hence the adjoint of $C_{r}$ has this cardinality because $c_{0}=C_{r} / C$. We have seen in the proof of Lemma 9 that each $w$-continuous $f$ on a Banach space $B$ is determined by a countable subset $K^{*}$ of $B^{*}$ in the sense that $f(x)=f(y)$ whenever $l(x)=l(y)$ for all $l \in K^{*}$. Moreover, if $g$ is the function on $B / K^{*}$ which is associated with $f$ (see the remark following Lemma 9) then $g$ is continuous, and there are at most $2^{N_{0}}$ such $g$ for each choice of $K^{*}$. This gives us the desired contradiction, since there are only $2^{\mathbb{N}_{0}}$ of the $K^{*}$.

Proof of (ii). If $C$ were a projection of $C_{r}$, then it is easy to check that $C_{r}$ would be isomorphic to $C \times c_{0}$. Hence $C_{r}$ in the weak topology would be homeomorphic to $C \times c_{0}$ in its weak topology. This is impossible because $C_{r}$ is not w-normal as we have just seen, while $C \times c_{0}$ is $w$-Lindelöf. $\left(C \times c_{0}\right.$ is isomorphic to the collection of all the functions which vanish at infinity on the union of two copies of $I$, one with the usual topology and one with the discrete topology.)

EXAmpLE 3. Let $X$ be the space of countable ordinals under the order topology. If $C_{0}(X)$ is as above, then there is a collection $\mathscr{K}$ of closed spheres in $C_{0}(X)$ with c.i.p. and with $\cap \Re=\varnothing$.

Proof. For each $\alpha \in X$, let $x_{\alpha}(\beta)=0$ if $\beta>\alpha$, and let $x_{\alpha}(\beta)=1$ if $\beta<\alpha$. Define $K_{\alpha}$ as the set of $x \in C_{0}(X)$ such that $\left\|x-x_{\alpha}\right\| \leqq 1 / 2$. Then $\cap\left\{K_{\alpha_{i}}: i=1,2, \cdots\right\} \neq \varnothing$, since $(1 / 2) x_{\beta}$ is in each $K_{\alpha_{i}}$ if $\beta \geqq \sup \left\{\alpha_{i}: i=1,2, \cdots\right\}$. However, if $z \in \cap \varkappa$, then $z(\alpha)=1 / 2$ if $\alpha>0$, as 
may be easily verified. Therefore, $z \in^{\prime} C_{0}(X)$, since $z$ does not vanish at infinity.

\section{REFERENCES}

1. R. H. Bing, Metrization of topological spaces, Canad. J. Math. vol. 3 (1951) pp. 178-186.

2. M. Bockstein, Un theoreme de separabilite pour les produits topologiques, Fund. Math. vol. 35 (1948) pp. 241-246.

3. H. J. Cohen, Sur un problime de M. Dieudonne, C. R. Acad. Sci. Paris vol. 234 (1952) pp. 290-292.

4. H. H. Corson, The determination of paracompactness by uniformities, Amer. J. of Math. vol. 80 (1958) pp. 185-190.

5. - Normality in subsets of product spaces, Amer. J. Math. vol. 81 (1959) pp. 785-796.

6. M. M. Day, Normed linear spaces, Berlin, Springer-Verlag, 1958.

7. L. Gillman and M. Jerison, Rings of continuous functions, New York, D. Van Nostrand, 1960.

8. A. Grothendieck Critères de compacticite dans les espaces fonctionnels gentraux, Amer. J. Math, vol. 74 (1952) pp. 168-186.

9. Paul R. Halmos, Measure theory, New York, D. Van Nostrand, 1950.

10. Irving Kaplansky, Functional analysis, New York, John Wiley, 1958.

11. John L. Kelley, General topology, New York, D. Van Nostrand, 1955.

12. Walter Rudin, Homogeneity problems in the theory of Cech compactifications, Duke Math. J. vol. 23 (1956), pp. 409-419.

13. T. Shirota, $A$ class of topological spaces, Osaka Math. J., vol. 4 (1953) pp. 23-40.

14. E. Szpilrajn, Sur denx proprietes des classes d'ensembles, Fund. Math. vol. 33 (1945). pp. 303-307.

15. Andre Weil, L'integration dans les groupes topologiques, Paris, Hermann, 1953.

UNIVERSITY OF WASHINGTON,

Seattle, Washington 